\title{
Use of Trochanteric Flip Osteotomy Improves the Outcome of Pipkin I and II Femoral Head Fractures
}

\author{
Dhakal RM¹, Shrestha RP², Shrestha B $^{3}$, Kandel IS ${ }^{3}$, Bista KB ${ }^{1}$, Tripathi ${ }^{1}$ \\ 'Lecturer, ${ }^{2}$ Professor, ${ }^{3}$ Associate Professor \& HOD, \\ Department of Orthopedics, Gandaki Medical College \& Teaching Hospital, Pokhara, Nepal
}

\begin{abstract}
Introduction: Annual incidence of femoral head fracture is constantly increasing due to increase in cases of road traffic accidents (RTA). Four to $17 \%$ of femoral head fractures are due to posterior dislocations of hip. Outcome of femoral head facture associated with posterior dislocation of hip, the pipkin I and II fracture, is unsatisfactory due to lack of universally accepted protocol for its management and treatment.
\end{abstract}

Objective: To evaluate the patient outcome using relatively newer approach, the Trochanteric Flip (digastrics) Osteotomy (TFO), for the precise anatomical reduction of femoral head fractures associated with posterior dislocation of hip.

Methods: Between 2013 and 2017, 21 patients with sustained isolated femoral head fracture were admitted at our hospital. We used TFO approach for the management and treatment of femoral head fractures. The patients were followed up for 42 months at different intervals. Clinical outcome were evaluated using Merle d'Aubigne Postel and Thompson-Epstein scale.

\section{Keywords}

Femoral head fracture,

Medial femoral circumflex artery,

Pipkin I and II,

Trochanteric flip osteotomy .

\section{Corresponding author}

*Dr. Rabi Mohan Dhakal, MS

Department of Orthopedics

Gandaki Medical College \& Teaching

Hospital, Pokhara, Nepal

Email:dhakalrabi@hotmail.com

\section{INTRODUCTION}

Femoral head fracture first described by Birkett in 1869 comprises a relatively rare injury ${ }^{1-4}$. Though uncommon, its incidence is constantly increasing due to annual increase in road traffic accidents (RTA) ${ }^{5}$. Four to $17 \%$ of femoral head fractures are due to posterior dislocations of hip ${ }^{4,6,7}$. Most common mechanism of this type of injury is dash board injury ${ }^{8}$. At present, there is no universally
Results: Retrospective analysis showed excellent, good, fair and poor results in five (23.8\%) patients, 13 (61.9\%) patients, two (9.5\%) patients and one (4.7\%) patient, respectively. Clinical outcomes included sciatic nerve injury (4.7\%), moderate arthritis (95.3\%), benign non-debilitating heterotrophic ossification (19\%), avascular necrosis of femoral head $(4.7 \%)$ and neuropraxia $\quad(4.7 \%)$.

Conclusions: Use of trochanteric flip osteotomy gives the favorable outcome for the treatment of this type of fracture. Most importantly, the vascularity of femoral head remains intact which makes TFO a very useful technique for pipkin I and II fracture treatment.

accepted protocol for the treatment of femoral head fracture. Treatment strategies may range from simple closed reduction, open reduction and internal fixation, prosthetic placement to excision of fractured segment ${ }^{6,9-11}$. Regardless of treatment approaches, serious long term consequences like post traumatic arthritis, avascular necrosis and heterotrophic ossification may complicate the natural course of healing and rehabilitation leading to unsatisfactory clinical outcome and variable degree 
of disability. Currently, an approach using trochanteric flip osteotomy (TFO) for anatomical reduction of the fracture has been shown to provide long term satisfactory outcome $e^{9,11-13}$.

Herein, we employed TFO for precise anatomical reduction and minimum vascular damage for pipkin type I and type II fracture.

The objective of this study is to validate an alternative newer technique to reduce pipkin type fracture for the better long term outcome with minimum perioperative and postoperative complications.

\section{METHODS}

\section{Cases}

Between June 2013 and Dec 2017, 21 patients; 16 male and five female with a mean age of 46.3 years (Range 18 - 67 years) with pipkin fracture underwent surgical treatment at our hospital. All the patients were associated with posterior hip dislocation. All of them were evaluated and treated by the attending orthopedics. Patients only with isolated femur head fracture (pipkin type I and type II) were included in the study. Patient with inadequate follow-up visits were excluded from the study.

\section{Surgical approach}

$\mathrm{X}$ ray and a computed tomography (CT) of hip joint were done for all patients (Fig 1). Definitive surgery was done within 72 hours except in one case in which the day of visit is at 60 after hip dislocation.

Fig 1: Pre-operative radiograph of a 37 year old female patient showing femur head fracture with pipkin type II fracture

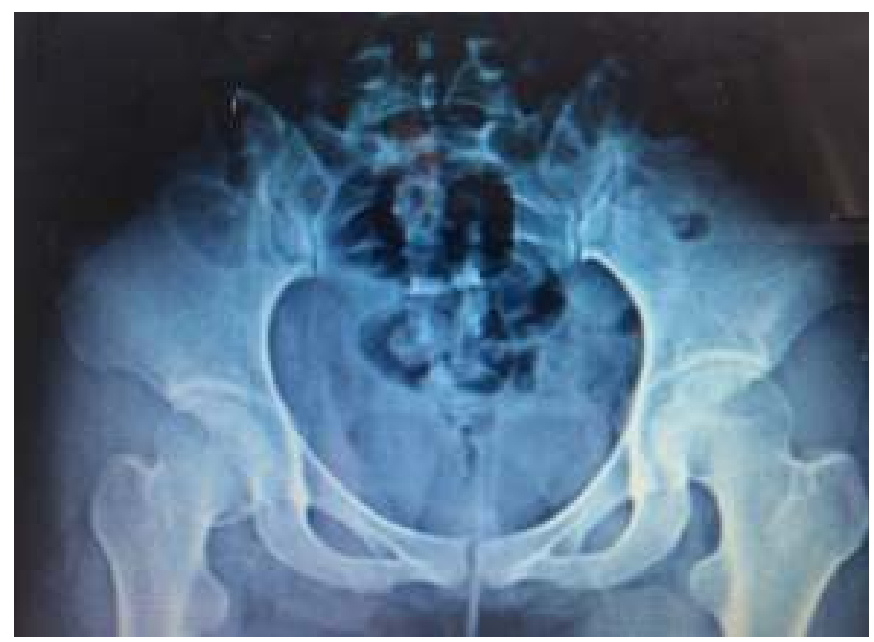

The average operative time was 90 minutes. All cases were operated in lateral decubitus position, posteriolateral Kocher langenbeck incision was made and the facia lata splitted accordingly. The leg was then internally rotated and the posterior border of gluteus medius identified. An incision was made from the posterosuperior edge of the greater trochanter extending distally to the posterior border of the ridge of vastus lateralis. Trochanteric flip osteotomy with maximum thickness of $1.3 \mathrm{~cm}$ was made along this line with an oscillating saw.

The osteotomy should exit just anterior to the most posterior insertion of gluteus medius to preserve and protect the profundus branch of the medial femoral circumflex artery (MFCA) which is the major blood suppy of the femur head ${ }^{1,8}$. The insertion of gluteus medius and vastus lateralis was preserved on the mobile trochanteric fragment. Then trochanteric fragment was mobilized and pushed interiorly. Joint capsule was exposed in the interval between piriformis and gluteus minimus. Great care was taken to avoid injury to the sciatic nerve which runs inferior to the piriformis muscle.

Z-shaped capsulotomy was done to visualize the hip joint. The capsulotomy remained anterior to the lesser trochanter to avoid damage to the main branch of the MFCA which is located just superior and posterior to lesser trochanter. This preserved the anastomosis between inferior gluteal artery and MFCA which runs along the distal border of the piriformis muscle and tendon ${ }^{8}$.

In order to dislocate the hip, the leg was flexed, externally rotated, brought over the front of the operating table and placed into a sterile bag. By manipulating the leg, acetabular cup and femoral head can be visualized in all directions. The stump of ligament teres can be removed from the head as the foveolar artery, which runs inside ligament teres, is not an important source of blood supply to the femoral head. After dislocation of the hip joint the acetabular cartilage and the labrum were carefully inspected for any damage.

The labrum was probed to inspect any labral tear. Small labral tear was debrided large tear was fixed with suture anchors. Detached fragment of the head was explored and put on the instrument table for later reassembly. Any soft tissue attachment on the fragment was preserved in order to avoid further devascularisation of these fragments. The detached fragment was brought into the best attainable relationship to the main fragment and secured with two 
to three Kirschner wires (Fig 2). Head fragment was fixed using $4 \mathrm{~mm}$ titanium cannulated screw over the wires. The wires were then removed followed by countersinking of the head of the screws below the cartilage level (Fig 3). Alternatively, Herbert screw or absorbable pins could be used ${ }^{1,2,14}$. C-arm fluoroscopy was used to confirm the stable fixation of the fracture.

Fig 2: Temporary fixation of fragments with k-wire and bone grafting

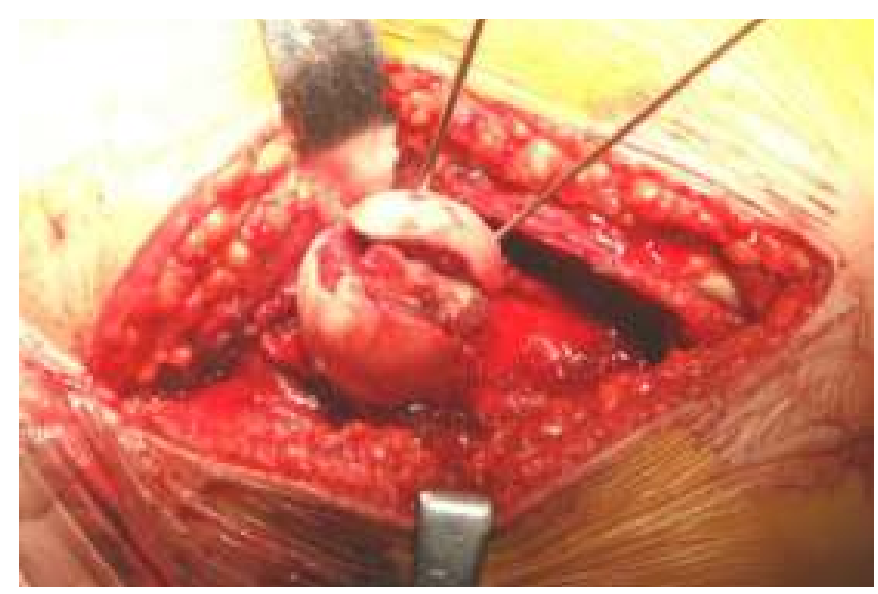

Fig 3: Fixation of head fragments with canulated screws

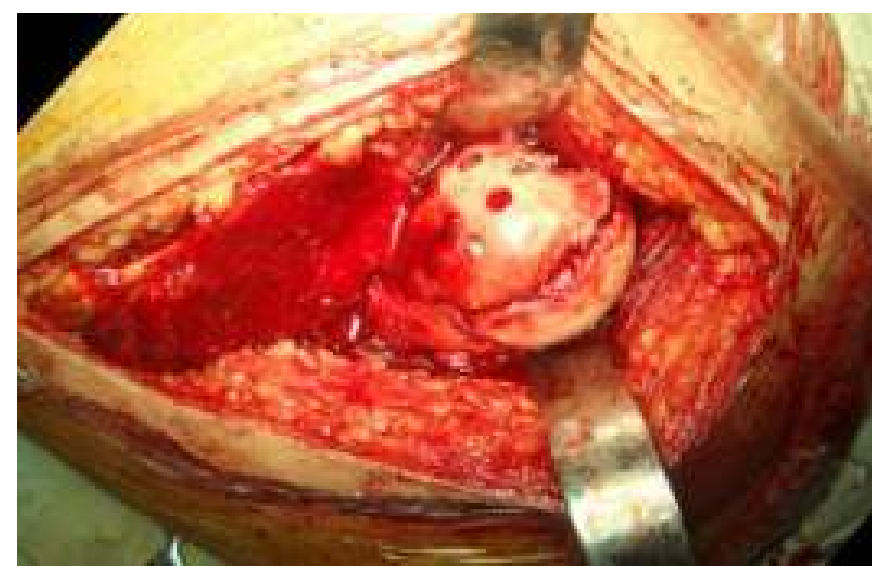

Little Bone graft was taken from the trochanteric osteotomy site to fill any gap between the bone fragment and femoral head. Acetabulum was carefully inspected for any bone fragment, torn pieces of capsule and synovial tissue. After fracture fixation the hip was successfully reduced by manual traction on the flexed knee and internal rotation. Capsule was loosely approximated by using 2-0 absorbable suture to avoid any tension that damage of retinacular vessel to avoid potential drop in femoral head perfusion. The trochanteric osteotomy was reduced and fixed by using two $4.5 \mathrm{~mm}$ cortical screws. Finally, C-arm $\mathrm{x}$-ray was done to confirm the screw placement. Drain was placed and wound was closed in layers.

\section{Perioperative and postoperative care}

All surgically treated patients received standard dose of perioperative antibiotics. Postoperatively, they received a course of nonsteroidal anti-inflammatory drugs to prevent heterotrophic ossification, and low molecular weight heparin for six weeks starting from second postoperative day to prevent deep vein thrombosis.

\section{Follow-up visit}

Average hospital stay was four days (Range from two to seven days). Duplex ultrasound was performed to rule out any deep venous thrombosis before discharge. After discharge, patients were followed clinically and radiographically (X-ray, CT-scan or MRI scan) at four weeks (Fig 4a), eight weeks (Fig 4b - 4d) and every three months thereafter. At each follow up patients were examined by the operating orthopaedic surgeon for active and passive range of motion, power, gait assessment, activity of daily living assessment, pain assessment and oblique radiograph of the operated hip. Only touchdown weight bearing was allowed for six weeks followed by gradual weight bearing. Gait training and full weight bearing was allowed eight weeks after surgery.

Fig 4: Post-operative radiographs of the 37 year old female patient with femur head fracture with pipkin type II fracture. (a) One month post-operative X-ray (b) 32 month post-operative X-ray (c) 32 month post-operative computed-tomography (CT) scan (d) 32 month postoperative magnetic resonance imaging (MRI) scan
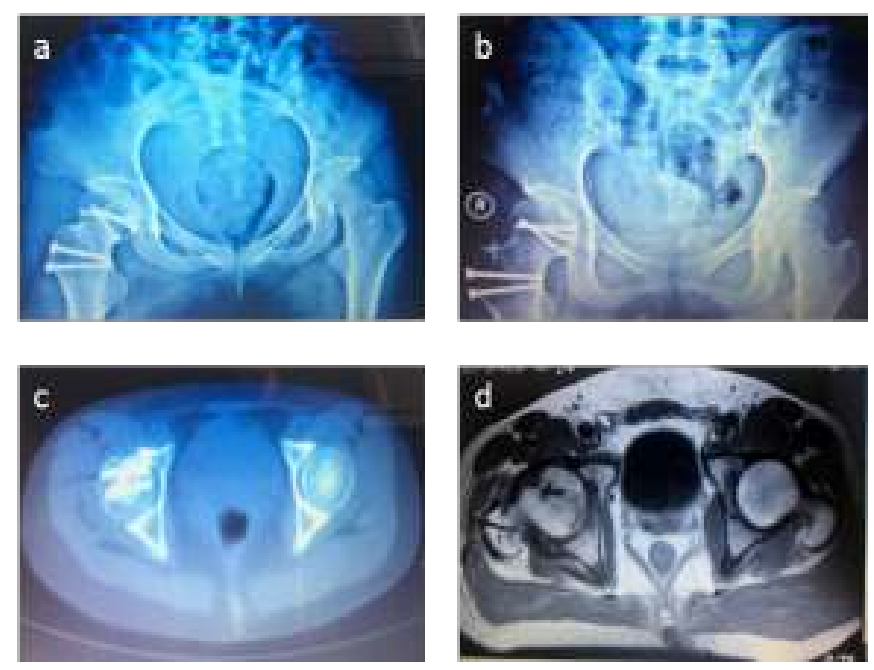

\section{Assessment of clinical outcome}

Clinical outcome scores were calculated by using Merle d' Aubigne-Postel and Thompson-Epstein scales. ThompsonEpstein scale is the measure for pain, function and radiographic appearance and is graded as excellent, good, 
fair or poor whereas Merle d' Aubigne Postel scale is a numeric scale with a maximum score of 18 and accounts for pain and functional outcome.

\section{RESULTS}

Among 21 patients with pipkin type I and type II fractures, 16 were males and five were females with a mean age 44.3 years age $(18-66)$. The patients were followed for 12 to 42 months (mean 29.6 months). Two patients were excluded from the study because of the inadequate followup (follow up less than 12 months).

The major cause of the injury was RTA (12) and the other were fall from height (Seven), and sports injury (Two). According to pipkin's classification, 14 cases were type I and remaining seven were type II. All of the cases with surgical dislocation were treated using TFO. None of them had an earlier traumatic or iatrogenic dislocation. Mean operating time from incision to closure was 90 minutes. The reduced fragments were within $2 \mathrm{~mm}$ in all operated cases as revealed by multiple radiographs and/ or postoperative CT scans. All the fractures were healed within 10 weeks. None of the cases showed trochanteric fixation defect.

The mean Merle d'Aubigne-postel score was 15.1 (Range 9 - 18) (Table 1). According to Thompson-Epstein criteria, five (23.8\%) patients had excellent, 13 (61.9.4\%) had good, two $(9.5 \%)$ patients had fair and one $(4.7 \%)$ patient had poor result (Fig 5).

Table 1: Summary of patient history and clinical outcome after TFO

$\begin{array}{ccccccccc}\text { Case } & \begin{array}{l}\text { Age } \\ \text { /sex }\end{array} & \text { Cause } & \begin{array}{l}\text { Pipkin } \\ \text { type }\end{array} & \begin{array}{l}\text { Compli- } \\ \text { cation }\end{array} & \begin{array}{l}\text { Follow-up } \\ \text { (months) }\end{array} & \text { TE } & \text { Mutcome } & \begin{array}{l}\text { Concurrent } \\ \text { injury }\end{array} \\ 1 & 55 / \mathrm{M} & \text { RTA } & \text { I } & & 36 & \text { Good } & 15 & \\ 2 & 60 / \mathrm{M} & \text { Fall } & \text { I } & & 30 & \text { Good } & 15 & \\ 3 & 47 / \mathrm{M} & \text { Fall } & \text { II } & & 38 & \text { Excellent } & 18 & \\ 4 & 33 / \mathrm{F} & \text { RTA } & \text { I } & \text { HO } & 31 & \text { Good } & 15 & \\ 5 & 45 / \mathrm{M} & \text { RTA } & \text { II } & & 24 & \text { Excellent } & 17 & \\ 6 & 58 / \mathrm{M} & \text { RTA } & \text { II } & \text { Mild PTA } & 27 & \text { Good } & 16 & \text { Rib } \\ 7 & 54 / \mathrm{M} & \text { Fall } & \text { II } & \begin{array}{l}\text { Nerve } \\ \text { injury }\end{array} & 20 & \text { Fair } & 13 & \text { fracture } \\ 8 & 67 / \mathrm{M} & \text { Fall } & \text { II } & \text { mild PTA } & 33 & \text { Good } & 15 & \\ 9 & 30 / \mathrm{F} & \text { RTA } & \text { I } & \text { HO } & 35 & \text { Good } & 15 & \\ 10 & 40 / \mathrm{M} & \text { Sports } & \text { I } & & 12 & \text { Excellent } & 18 & \\ 11 & 41 / \mathrm{M} & \text { Fall } & \text { I } & \text { Moderate } & 18 & \text { Fair } & 14 & \\ 12 & 55 / \mathrm{F} & \text { RTA } & \text { II } & & 23 & \text { Good } & 17 & \\ 13 & 59 / \mathrm{M} & \text { Fall } & \text { I } & \text { Mild PTA } & 28 & \text { Good } & 16 & \\ 14 & 37 / \mathrm{F} & \text { Fall } & \text { II } & \text { HO } & 32 & \text { Good } & 16 & \\ 15 & 42 / \mathrm{M} & \text { RTA } & \text { II } & & 38 & \text { Excellent } & 17 & \\ & & & & & & & & \end{array}$

\begin{tabular}{lllllllll}
16 & $20 / M$ & $\begin{array}{l}\text { Sports } \\
\text { injury }\end{array}$ & I & & 36 & Good & 16 & $\begin{array}{c}\text { Humerus } \\
\text { fracture }\end{array}$ \\
17 & $18 / \mathrm{M}$ & RTA & I & HO & 24 & Good & 17 & \\
18 & $39 / \mathrm{M}$ & Fall & II & & 27 & excellent & 18 & \\
19 & $40 / \mathrm{F}$ & RTA & II & PTA & 28 & Good & 15 & \\
20 & $66 / \mathrm{M}$ & RTA & I & & 41 & Good & 15 & \\
21 & $26 / M$ & Fall & I & AVN & 42 & Poor & 9 & $\begin{array}{c}\text { Head } \\
\text { injury }\end{array}$ \\
\hline
\end{tabular}

TE: Thompson and Epstein, MA: Marle d'Aubigne Postel, PTA: Post Traumatic Arthritis, HO: Heterotrophic Ossification, AVN: Avascular Necrosis of femoral head

Fig 5: Pie chart showing the clinical outcome after TFO therapeutic approach in 21 patients

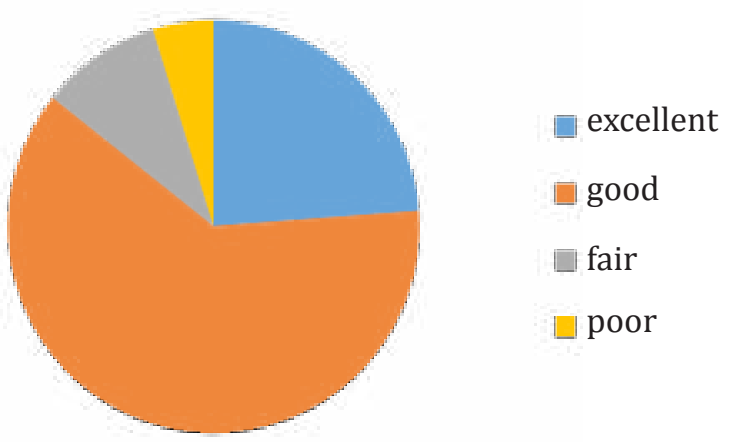

Six out of 21 (28.5\%) developed mild to moderate level of post traumatic arthritis (PTA). Heterotrophic ossification (HO) was seen in four (19\%) patients. None of the patients developed hip ankylosis. One (4.7\%) patient developed partial neurapraxia of sciatic nerve after operation. Six months after the surgery the patient showed gradual recovery of the nerve injury. There was complete recovery of the injury within nine months as proved clinically and by nerve conduction studies. One (4.7\%) patient developed avascular necrosis of femoral head.

\section{DISCUSSION}

Femur head fracture results from traumatic injury, and most frequently associated with posterior hip dislocation ${ }^{10,13,15}$. Available literature reported good or excellent outcome only in 40 to $70 \%$ of patients who underwent TFO therapy ${ }^{5,9,10,15,12,16}$. Herein, we adopted TFO therapeutic protocol for patients with femur head fracture with posterior hip dislocation and assessed the clinical outcome.

Femoral head fracture with posterior dislocation represents a few of the orthopedic emergencies. Several authors agreed that early reduction leads to better outcome than the reduction delayed by more than 24 hours. Relationship between delayed reduction and 
increased incidence of femoral head osteonecrosis is well established $\mathrm{d}^{7,12,17}$.

Treatment of femoral head fracture poses a potential risk of iatrogenic femoral neck fracture or necrosis. Potential iatrogenic femoral neck fracture was avoided by giving good muscle relaxants. Moreover, to avoid iatrogenic damage anatomical knowledge of course and location of the deep branch of MFCA is very important. Principal blood supply for the femoral head, particularly the weight bearing portion is from the terminal branch of the MFCA ${ }^{11,18}$. Medial epiphyseal artery through the ligamentum teres supplies only the perifoveal area and rarely supplies significant portion of the head. Lateral femoral circumflex artery has little or no contribution to the femoral head blood supply ${ }^{11,18}$. Deep branch of MFCA, a branch of profunda femoris artery, travels posteriorly and lies outside the capsule towards the intertrochanteric crest between iliopsoas laterally and pectineus medially along the base of femoral neck. Trochanteric branch that rises adjacent to the proximal border of quadrates femoris, travels across the lateral surface of the greater trochanter. Posterior to the tendon of obturator externus passes the main vessels that perforates the capsule of hip joint slightly superior to the insertion of the superior gamellous tendon and divides into several terminal branches which enter the femoral head just lateral to the articular junction ${ }^{10,19}$. Deep branch of MFCA can easily be damaged during the posterior approach to the hip joint resulting primary loss of blood supply to the femoral head. However, the approach, the greater trochanteric flip osteotomy, performed in this study helps to preserve the short external rotators. Intact short external rotators protect the deep branch of MFCA thus helps to avoid the femoral head necrosis.

Treatment of these types of injuries is complex and should be dealt by expert surgeon. Different surgical approaches have been used for the treatment of femoral head fractures including anterior (Smith Patterson), anterolateral (Watson Jones), lateral, medial (ludloff), posterior (Kocher langenbeck), modified posterior (Trochanteric flip osteotomy). With anterior approach femoral head dislocation can be performed but the exposure is much limited and the acetabulum cannot be inspected fully. It also makes the screw fixation more difficult. Similarly anterolateral and direct lateral approach also allow the dislocation of femoral head but the drawback of these approaches is difficult and incomplete view of acetabulum.
In classical posterior approach, tenomyotomy of external rotator muscles affect anastomosis of inferior gluteal artery and deep branch of MFCA which lead to high occurrence of femoral head necrosis. Also the reattachment of external rotator muscles may not be stable.

According to Ganz, trochanteric flip osteotomy is a safe method for the surgical dislocation of the femur head ${ }^{10,19}$. We chose TFO for the surgical reduction of pipkin I and II fractures as it allows surgeons to view femur head and acetabulum completely. Also, the posterior wall, femur head fracture and the dome area are well exposed with this technique. This enabled precise reduction of the fracture.

With posterior approach, the abductors would be forcefully retracted to view dome area which may cause loss of abductor strength. However, TFO approach allowed easy dislocation with no detachment of external rotator muscle, so that the abductor strength is preserved. ${ }^{20,21}$.

The scoring systems we used are Merle d'aubigne- postel and Thompson Epstein. With little modification of already established surgical dislocation of hip (see methods), we received clinically excellent outcome compared to the previous findings (40 - 70\% compared to $86 \%$ in this study) $)^{5,9,10,12,15,16}$.

In pipkin fracture there is usually significant gap between the fractured segments because of destruction of cartilage and cancellous bone at the region of fovea. Since the outcome of this type of fracture significantly depends upon the level of anatomical reduction of the fractured segments, role of autograft is very important to accelerate the healing of fracture. The firm packing of the gap between the fractured segments with the use of autograft is not only the important step to accelerate the bone healing but also the necessary step to reduce severe post-operative arthritis. This helps to smooth the hip joint movement. With other approach bone graft can't be taken from single-stage surgery. However, TFO allowed us to obtain the necessary amount of bone graft from the osteotomy site avoiding multi-stage surgery as seen with other approaches.

In our procedure, we used stainless steeland titanium screw to fix the bone fragments. With the use of metal screw we can achieve better fixation of the bone fragments. The lag effect of the metal screw is found to be stronger than that 
of absorbable. Previous studies reported adverse tissue reaction to fixation implants made of polyglycolic acid ${ }^{14,22}$. With the use of metal implants none of the cases were reported to have adverse tissue reaction. In five cases we used titanium screw to reduce the cost of surgery. Because of this we couldn't perform follow up MRI in those cases which is very important and relevant diagnostic tool to diagnose the femur head necrosis. In those cases we relied on plane X-ray, CT scan and PET scan to see the status of femur head necrosis. Important recommendation is to use titanium screw for the fixation of both head fragment and the trochanteric fragments.

Avascular necrosis is a major complication which occurs due to extra-capsular and extra-osseous injury to nutrient vessels $^{1,6,23}$. Depending on the severity of trauma to the hip and time span after the traumatic dislocation, avascular necrosis of femur head is more likely. In TFO external rotator muscle is not interrupted which in turn protects MFCA. Thus reducing the chances of necrosis and allowing MFCA to perfuse femoral head adequately. Considerable amount of blood loss occurs from drill hole in femoral head. Postoperative assessment by using MRI scan suggests adequate perfusion. None of the cases in our study showed avascular necrosis during their follow-up.

Heterotrophic ossification following fracture of the femoral head is quite common; reported incidence varies from six to $64 \%{ }^{12,24-26}$. The potential risk is due to aggressive muscular striping from the ileum during the approach. Anterior approach to the hip has been reported to be risk factor for the development of the heterotrophic ossification ${ }^{27}$. With TFO approach the mobilization of the muscles from ileum is much less than any previous approaches resulting less case of heterotrophic ossification.

Minimum complications occur if TFO is done keeping the landmark in view. Sciatic nerve injury occurs if care is not taken as the sciatic nerve lies beneath the piriformis and proximal to the external rotator muscle. One of the case developed neurapraxia of the sciatic nerve. However, in that particular patient definitive surgery was done 60 days after the injury. Because of such a long delay in visit to the centre, there was adherence of the nerve. We presume that mobilisation of the adhered nerve during the surgical procedure was most likely the cause of neurapraxia. This could be minimized with timely surgical intervention. In delayed cases, careful surgical dissection and adequate exposure of the sciatic nerve before dislocation of the femur head was suggested. This is one of the crucial steps to avoid iatrogenic injury to the nerve. Moreover, after the exposure of the nerve it can be secured under piriformis muscle. Care should be taken at placing the retractors at the time of traction. Also, post-operative rehabilitation and physical therapy is also an important step for the timely recovery of the injured nerve. In our typical case, the nerve function returned to normal after six months.

Trochanteric non-union which is one of the commonly reported complication of the classical trochanteric osteotomy is a rare complication with TFO as digastrics muscle helps stabilize the trochanteric fragment. Also, the gluteus minimus is manipulated under vision and excessive retraction of the gluteus midius is not required, the heterotrophic ossification is much less with TFO.

The limitation of the study is relatively few number of cases due to rarity of the fracture. However, the common surgical approach used showed promising result for management and treatment of femur head fracture by TFO. To better understand the clinical outcome of the procedure, a large multi-centre study is needed.

\section{CONCLUSIONS}

In conclusion, TFO allows complete view of femur head and actabulum. It leaves MCFA intact reducing avascular necrosis and hypertrophic ossification. Moreover, labral and cartilaginous pathology which were unclear on MRI can be easily viewed during this surgical technique. Most importantly, the vascularity of the femur head remains intact which makes TFO a very useful technique for treatment of pipkin I and II fracture, and even in cases with delayed surgical interventions, if measures to prevent sciatic nerve injury were taken in consideration.

\section{Conflict of Interest}

The authors do not have any conflict of interest to declare.

\section{REFERENCES}

1. Guo JJ, Tang N, Yang HL, Qin L, Leung KS. Impact of surgical approach on postoperative heterotopic ossification and avascular necrosis in femoral head fractures: A systematic review. Int Orthop. 2010. 34(3): 319-22. 
2. Solberg BD, Moon CN, Franco DP. Use of a trochanteric flip osteotomy improves outcomes in Pipkin IV fractures. Clin Orthop Relat Res. 2009. 467(4): 929-33.

3. Harrahill M. Posterior hip dislocation with femoral head fracture: An unusual injury. J Emerg Nurs. 2006; 32(5): 451-3.

4. Droll KP, Broekhuyse H, O'Brien P. Fracture of the femoral head. J Am Acad Orthop Surg. 2007; 15(12): 716-27.

5. Oransky M, et al. Fractures of the femoral head: A long-term follow-up study. Musculoskelet Surg. 2012; 96(2): 95-9.

6. Yue JJ, et al. Posterior hip dislocations: a cadaveric angiographic study. J Orthop Trauma. 1996; 10(7): 447-54.

7. Brumback RJ, et al. Fractures of the femoral head. Hip.1987; 181-206.

8. Jukkala-Partio $\mathrm{K}$, et al. Absorbable fixation of femoralhead fractures. A prospective study of six cases. Ann Chir Gynaecol. 1998; 87(1): 44-8.

9. Dreinhofer KE, et al. Femur head dislocation fractures. Long-term outcome of conservative and surgical therapy. Unfallchirurg. 1996; 99(6): 400-9.

10. Gautier E, et al. Anatomy of the medial femoral circumflex artery and its surgical implications. $J$ Bone Joint Surg Br. 2000; 82(5): 679- 83.

11. Sevitt S, Thompson RG. The Distribution and Anastomoses of Arteries Supplying the Head and Neck of the Femur. J Bone Joint Surg Br. 1965; 47: 560-73.

12. Hougaard K, Thomsen PB. Traumatic posterior dislocation of the hip--prognostic factors influencing the incidence of avascular necrosis of the femoral head. Arch Orthop Trauma Surg. 1986; 106(1): 32-5.
13. Stewart MJ, Milford LW. Fracture-dislocation of the hip; an end-result study. J Bone Joint Surg Am. 1954; 36(A:2): 315-42.

14. Prokop A, et al. Biodegradable implants for Pipkin fractures. Clin Orthop Relat Res. 2005; (432): 22633.

15. Kelly RP, Yarbrough SH. 3rd, Posterior fracturedislocation of the femoral head with retained medial head fragment. J Trauma. 1971; 11(2): 97108.

16. Marchetti ME, Steinberg GG, Coumas JM. Intermediate-term experience of Pipkin fracturedislocations of the hip. J Orthop Trauma. 1996; 10(7): 455-61.

17. Epstein HC, Wiss DA, Cozen L. Posterior fracture dislocation of the hip with fractures of the femoral head. Clin Orthop Relat Res. 1985; (201): 9-17.

18. Trueta J. The normal vascular anatomy of the human femoral head during growth. J Bone Joint Surg Br. 1957; 39-B(2): 358-94.

19. Ganz R, et al. Surgical dislocation of the adult hip a technique with full access to the femoral head and acetabulum without the risk of avascular necrosis. J Bone Joint Surg Br. 2001; 83(8): 1119-24.

20. Nutton RW, Checketts RG. The effects of trochanteric osteotomy on abductor power. J Bone Joint Surg Br. 1984; 66(2): 180-3.

21. Amstutz HC, Maki S. Complications of trochanteric osteotomy in total hip replacement. J Bone Joint Surg Am. 1978; 60(2): 214-6.

22. Bostman OM, Pihlajamaki HK. Adverse tissue reactions to bioabsorbable fixation devices. Clin Orthop Relat Res. 2000; (371): 216-27.

23. Giannoudis PV, et al. Management, complications and clinical results of femoral head fractures. Injury. 2009; 40(12): 1245-51. 
24. Lang-Stevenson A, Getty CJ. The Pipkin fracturedislocation of the hip. Injury. 1987; 18(4): 264-9.

25. Swiontkowski MF, Thorpe M, Seiler JG, Hansen ST. Operative management of displaced femoral head fractures: case-matched comparison of anterior versus posterior approaches for Pipkin I and Pipkin II fractures. J Orthop Trauma. 1992; 6(4): 437-42.

26. Peter Kloen, Klaus A. Siebenrock, Ernst L. F. B. Raaymakers, Rene K. Marti, Reinhold Ganz. Femoral Head Fractures Revisited. European Journal of Trauma. 2002; 28( 4): 221-233. 